\title{
Modulation of the Receptive Fields of Midbrain Neurons Elicited by Thalamic Electrical Stimulation through Corticofugal Feedback
}

\author{
Yamin Wu and Jun Yan \\ Department of Physiology and Biophysics, Hotchkiss Brain Institute, Faculty of Medicine, University of Calgary, Calgary, Alberta, Canada T2N 4N1
}

\begin{abstract}
The ascending and descending projections of the central auditory system form multiple tonotopic loops. This study specifically examines the tonotopic pathway from the auditory thalamus to the auditory cortex and then to the auditory midbrain in mice. We observed the changes of receptive fields in the central nucleus of the inferior colliculus of the midbrain evoked by focal electrical stimulation of the ventral division of the medial geniculate body of the thalamus. The receptive field of an auditory neuron was characterized by five parameters: the best frequency, minimum threshold, bandwidth, size of receptive field, and average spike number. We found that focal thalamic stimulation changed the parametric values characterizing the recorded collicular receptive fields toward those characterizing the stimulated thalamic receptive fields. Cortical inactivation with muscimol prevented the development of the collicular plasticity induced by focal thalamic stimulation. Our data suggest that the intact colliculo-thalamo-cortico-collicular loops are important for the coordination of sound-guided plasticity in the central auditory system.
\end{abstract}

Key words: auditory; thalamocortical; corticocollicular; neural plasticity; descending system; mouse

\section{Introduction}

Auditory learning and experience induce sound-specific plastic changes in the receptive fields (RFs) of neurons in the auditory cortex and subcortical auditory nuclei (Gonzalez-Lima and Agudo, 1990; Gao and Suga, 1998, 2000; Weinberger, 1998). Furthermore, the plasticity is similar in terms of RF changes in both the auditory cortex and subcortical auditory nuclei; the RFs shift toward the frequency of the acquired sound. The occurrence of the sound-specific plasticity induced by auditory learning and/or experience has been classically interpreted as the result of neural interactions in the ascending auditory system. For example, learning-induced auditory plasticity is believed to begin at the auditory thalamus and then further develops in the auditory cortex (Weinberger, 1998). However, the explanations provided by such an ascending model cannot adequately account for the fact that a cortical application of a muscarinic receptor antagonist eliminates the learning-induced plasticity in the auditory cortex and reduces the plasticity in the auditory midbrain (Ji et al., 2001; Ji and Suga, 2003).

Studies over the past decade reveal that the corticofugal system mediates frequency-specific neural plasticity in the auditory midbrain and thalamus (Zhou and Jen, 2000; Yan and Ehret,

Received March 23, 2007; revised July 8, 2007; accepted Aug. 13, 2007.

This work was supported by the National Sciences and Engineering Research Council of Canada, the Campbell McLaurin Chair for Hearing Deficiencies of the University of Calgary, and Canadian Institutes of Health Research Grant MOP 82773.

Correspondence should be addressed to Jun Yan, Department of Physiology and Biophysics, Hotchkiss Brain Institute, Faculty of Medicine, University of Calgary, 3330 Hospital Drive Northwest, Calgary, Alberta, Canada T2N 4N1.E-mail: juyan@ucalgary.ca.

DOI:10.1523/JNEUROSCI.1320-07.2007

Copyright $\odot 2007$ Society for Neuroscience $\quad 0270-6474 / 07 / 2710651-08 \$ 15.00 / 0$
2002; Suga and Ma, 2003). Other studies using auditory fear conditioning or electrical stimulation of the cholinergic basal forebrain paired with tonal stimulation have established that the frequency-specific plasticity of subcortical RFs is mostly dependent on the corticofugal system (Gao and Suga, 1998; Ji et al., 2001; Ma and Suga, 2003; Zhang et al., 2005). Both the ascending and descending (corticofugal) pathways are apparently involved in learning-induced or experience-dependent neural plasticity in the auditory system (Suga et al., 2002; Suga and Ma, 2003; Yan, 2003). Anatomical and physiological studies indicate that the ascending and descending projections form multiple feedback loops that are tonotopically organized (Redies et al., 1989; Winer et al., 1999; Winer et al., 2001; Takayanagi and Ojima, 2006; Lim and Anderson, 2007). Do the tonotopic loops allow RF plasticity to be achieved and coordinated at different levels of the auditory system?

We have proposed that the tonotopic loops are critical circuitry responsible for the sound-specificity of learning-induced or experience-dependent neural plasticity in the central auditory system (Yan, 2003). Our previous work has demonstrated that focal electrical stimulation (ES) of the ventral division of the medial geniculate body $(\mathrm{MGBv})$ of the thalamus $\left(\mathrm{ES}_{\mathrm{MGB}}\right)$ induces centripetal plasticity of cortical RFs in multiple parameters including best frequency, minimum threshold, bandwidth, RF size, and response magnitude (Jafari et al., 2007). If the tonotopic loops coordinate the plasticity at different levels of the central auditory system, $\mathrm{ES}_{\mathrm{MGB}}$ should induce similar plasticity in the central division of the inferior colliculus (ICc) of the midbrain. Our results show that the $\mathrm{ES}_{\mathrm{MGB}}$ induces highly specific plasticity of collicular RFs similar to that of the auditory cortex and that a 
cortical application of muscimol, a $\mathrm{GABA}_{\mathrm{A}}$ agonist, completely abolishes this collicular plasticity.

\section{Materials and Methods}

Animal preparation. Thirty C57 female mice aged 5-9 weeks and weighing 17.1-24.6 g were used in this study. All experimental procedures were in accordance with the Canadian Council on Animal Care and approved by the Animal Care Committee of the University of Calgary. Animals were anesthetized with a mixture of ketamine $(100 \mathrm{mg} / \mathrm{kg}$, i.p. $)$ and $x y-$ lazine $(10 \mathrm{mg} / \mathrm{kg}$, i.p.) during surgery. The anesthetic status was confirmed approximately every $30 \mathrm{~min}$ by pinching the animal's tail with forceps. If the animal exhibited any response to tail pinching, additional dosages of ketamine $(20 \mathrm{mg} / \mathrm{kg})$ and xylazine $(2 \mathrm{mg} / \mathrm{kg})$ were administered to maintain anesthesia. The mouse head was fixed in a custommade head holder by rigidly clamping it between the palate and nasal/ frontal bones. The angle of the head was adjusted, so that bregma and lambda points lay in the horizontal plane. The scalp and temporal muscles were then removed to expose the left skull. A dental drill was used to make three openings in the left skull. One opening exposed the auditory cortex for applying physiological saline or muscimol solution and a second opening was made $5.02-5.34 \mathrm{~mm}$ caudal to the bregma and $0.5-2.0$ $\mathrm{mm}$ left of the midline for a penetration of recording electrodes in the ICc. A third opening was made $3.1 \mathrm{~mm}$ posterior to the bregma and 1.9 $\mathrm{mm}$ left of the midline for placing the electrical stimulation electrode in the MGBv (Franklin and Paxinos, 1996). After surgery, the animal was placed in a soundproof chamber for electrophysiological experiments. The animal's body temperature was maintained at $\sim 37^{\circ} \mathrm{C}$ by a feedback controlled heating pad.

Acoustic stimulation. The acoustic stimulus was a $60 \mathrm{~ms}$ tone burst with $5 \mathrm{~ms}$ rise/decay times. Sinusoidal waves were first digitally generated and then converted into 20 -volt peak-to-peak analog signals by a RP2.1 realtime processor (Tucker-Davis Technologies, Alachua, FL). To deliver the tone burst to the animal, the signals were fed to a tweeter via a digital attenuator (PA5) and power amplifier. The tweeter was placed $45^{\circ}$ to the right of and $90 \mathrm{~cm}$ away from the mouse right ear. The tone amplitude, expressed as dB sound-pressure level (SPL; reference pressure of $20 \mu \mathrm{Pa}$ ), was calibrated at the position of the animal's right ear with a LarsonDavis (Dalimar Instruments, Vaudreuil-Dorin, Quebec, Canada) condenser microphone (model 2520). The frequency and intensity of the tone burst were varied manually or computer controlled.

The excitatory responses of auditory neurons to acoustic stimuli were recorded with a computer-controlled frequency/amplitude (FA) scan (frequency-scan plus amplitude-scan). The receptive fields and/or frequency-threshold curves (frequency-tuning curves) were derived from the FA-scan data. In the FA-scan generated by BrainWare software (Tucker-Davis Technologies), the tone frequency was from 3 to $40 \mathrm{kHz}$ in $1 \mathrm{kHz}$ steps and the tone amplitude was from $103 \mathrm{~dB}$ SPL to $-2 \mathrm{~dB}$ SPL in $5 \mathrm{~dB}$ steps. Therefore, a FA-scan consisted of 799 frequency/amplitude blocks. The last block without tone presentation was designed for spontaneous spike counting. The interval between tone burst stimuli was 250 ms. The identical stimulus was repeated five times for each frequency/ amplitude set.

Recording of action potential and electrical stimulation in the MGBv. The stereotaxic coordinates of the MGBv are $3.1 \mathrm{~mm}$ posterior to the bregma, $1.9 \mathrm{~mm}$ lateral to the midline and $\sim 2.8 \sim 3.0 \mathrm{~mm}$ below the brain surface (Franklin and Paxinos, 1996). A tungsten electrode of $\sim 2-\mathrm{M} \Omega$ impedance was dorsoventrally inserted into the MGBv according to the above stereotaxic coordinates. The electrode was initially connected to the preamplifier of the recording system (see recording of ICc neurons below). A tone burst was delivered once per second during an electrode penetration and the tone frequency and amplitude were manually varied. Once a neuron responding to a tone stimulus was found, the electrode penetration was continued until there were no recorded auditory responses. This point represented the ventral boundary of the medial geniculate body. The electrode was slowly withdrawn by $50-100 \mu \mathrm{m}$ and the responses of thalamic neurons to the FA-scan were recorded. If the recorded neurons were not sharply tuned to a particular frequency, the electrode was withdrawn and another electrode penetration was made until sharply tuned neurons were recorded. This method ensured that our electrode tip was within the MGBv as supported by the histological examination of the lesion mark in our previous study (Jafari et al., 2007). The sharply tuned neurons were often found when the electrode tip was $\sim 2.8 \sim 3.0 \mathrm{~mm}$ below the brain surface. The electrode was then kept in position, but its connection was switched from the recording system to the electrical stimulation system. This procedure ensured that the locations of the recording and stimulating sites were identical.

Single electrical pulses $(0.2 \mathrm{~ms}$, monophasic and negative constant current square wave) generated by a Grass S88 stimulator (AstroMedical, West Warwick, RI) and an A360 constant-current isolator (World Precision Instruments, Sarasota, FL) provided the electrical stimulation. The output current of the isolator was set at $700 \mathrm{nA}$. The negative pole of the isolator was connected to the tungsten-wire electrode and the positive pole was connected to a clip on the scalp. The electrical stimulation was delivered at a rate of $4 \mathrm{~Hz}$ for $30 \mathrm{~min}$.

Recording of the auditory responses of ICc neurons. The auditory responses of two ICc neurons were simultaneously recorded with two separate tungsten-wire electrodes of $\sim 2-\mathrm{M} \Omega$ impedance. Sharply tuned neurons were typically recorded when the electrode tips were at a depth between 200 and $1800 \mu \mathrm{m}$ below the dorsal surface of the inferior colliculus. Action potentials recorded with two electrodes were fed to and digitized by a RA16PA multichannel preamplifier. Its output was connected to a RA16 Medusa multichannel amplifier with an optic fiber cable (Tucker-Davis Technologies). The RA16 bandpass filter was set to $0.3-3 \mathrm{kHz}$. Action potentials (spikes) were amplified 10,000 times and were selected by positive and negative level triggers. Spike waveforms were archived with BrainWare (Tucker-Davis Technologies) based on eight parameters of the action potential waveform including peak, valley, spike height, spike width, peak time, valley time, and two selected voltage values (Yan and Ehret, 2002; Zhang et al., 2005; Jafari et al., 2007).

Once the action potential recording was stable, the auditory responses of collicular neurons to the FA-scan were recorded and saved as control responses. Five minutes after an application of physiological saline or muscimol solution to the auditory cortex (see next section), the neuronal responses to the FA-scan were recorded again. These data were used as control data for electrical stimulation of the MGBv. The electrical pulses (4 per second for $30 \mathrm{~min}$ ) were then delivered to the MGBv neurons, which had been electrophysiologically studied. After the 30 min electrical stimulation of the MGBv neurons, the responses of ICc neurons to the FA-scan were recorded every 30 min until the $\mathrm{ES}_{\mathrm{MGB}}$-induced changes of ICc RFs recovered by at least $50 \%$.

Cortical application of saline or muscimol solution. Muscimol was used to inhibit the primary auditory cortex (AI) and to assess corticofugal involvement of collicular RF plasticity. The AI was first electrophysiologically located by examining the distinction of best frequencies at 4-7 cortical sites (Zhang et al., 2005). The dura covering, the AI was removed. A polythene tubing (1.5 $\mathrm{mm}$ in inner diameter and $1 \mathrm{~mm}$ in length) with a small amount of Vaseline at one end was gently placed on the surface of the brain to form a well which covered the AI. Muscimol solution (1 $\mathrm{mg} / \mathrm{ml}$, dissolved in $0.9 \%$ saline; Sigma, St. Louis, MO) or $0.9 \%$ saline was dropped into the well with a $1.0 \mathrm{ml}$ syringe immediately after obtaining the control data, the responses of MGBv and ICc neurons to the FA-scan. The responses of the MGBv and ICc neurons to the FA-scan were again recorded $5 \mathrm{~min}$ after a muscimol or saline application. The $\mathrm{ES}_{\mathrm{MGB}}$ started $25 \mathrm{~min}$ after the cortical application of muscimol or saline solution.

Data acquisition and processing. When the excitatory responses of the MGBv neurons at the stimulation site and the ICc neurons at the recording site to the FA-scan were recorded as the control, either saline or muscimol solution was applied to the AI and the responses of ICc neurons to the FA-scan were recorded again as the control of $\mathrm{ES}_{\mathrm{MGB}}$. The electrode placed in the MGBv was switched to the stimulating system. From this point onwards, the MGBv neurons were electrically stimulated and ICc RFs were recorded every $30 \mathrm{~min}$ until the $\mathrm{ES}_{\mathrm{MGB}}$-induced changes of ICc RFs recovered by at least $50 \%$.

Single-unit responses to the FA-scans were displayed by dot raster and peristimulus time (PST) histograms with a bin width of $1.0 \mathrm{~ms}$. The RF was the excitatory response area encompassed by the frequency-tuning curve that was derived from the FA-scan data. The response threshold at 
A

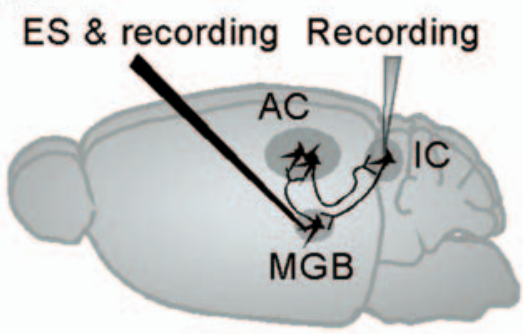

C

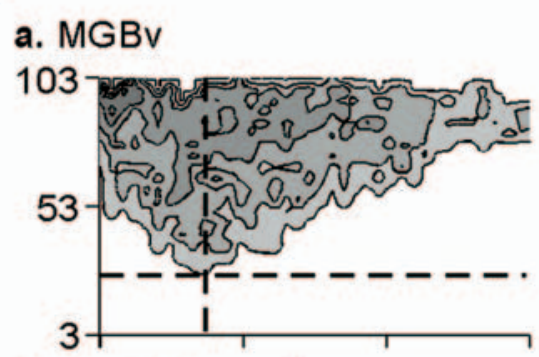

\section{b. ICc before saline}



c. ICc after saline

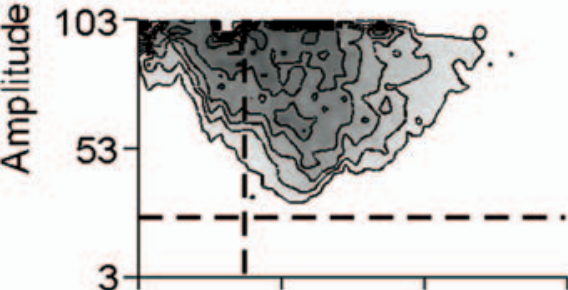

d. ICc after ESMGB

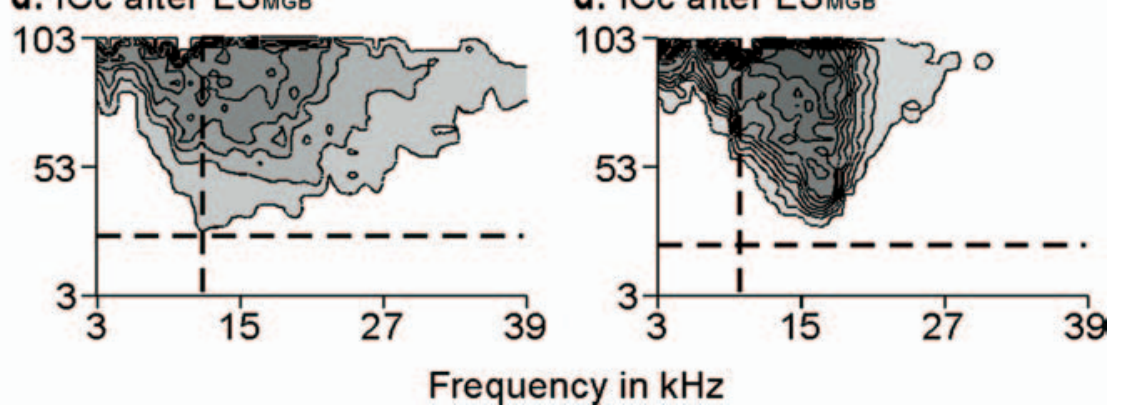

Figure 1. $A$, A dorsolateral view of the mouse brain as seen from the left side and schematic drawings of the inferior colliculus (IC), MGB, and auditory cortex (AC) as well as the colliculothalamic, thalamocortical, and corticocollicular projections. $B$, PST histograms displaying the responses of a stimulated MGBv neuron to a tone at its BF ( $18 \mathrm{kHz})$ and $20 \mathrm{~dB}$ above its MT (43 dB SPL) before $(\boldsymbol{a})$ and after $(\boldsymbol{b}) \mathrm{ES}_{\mathrm{MGB}}$. $\boldsymbol{C}$, The RFs of the stimulated MGBv neuron $(\boldsymbol{C} \boldsymbol{a})$ and recorded ICc neuron $(\boldsymbol{C} \boldsymbol{b}-\boldsymbol{C} \boldsymbol{d})$. A cortical application of saline did not change ICC RF $(\boldsymbol{C} \boldsymbol{C})$, but $E S_{M G B} \operatorname{did}(\boldsymbol{C d})$. D , The RFs of the stimulated MGBv neuron (Da) and recorded ICc neuron ( $\boldsymbol{D} \boldsymbol{b}-\boldsymbol{D} \boldsymbol{d})$. They were different from the MGBv and ICc neurons shown in $\boldsymbol{C}$. A cortical application of muscimol slightly changed the ICC RF (Dc). $E_{M G B}$ had little effect (Dd). The contour lines are drawn at 20,40,60, and $80 \%$ of the maximum spikes. ES, Electrical stimulation. The vertical and horizontal dashed lines represent the control BFs and MTs of the stimulated thalamic neurons.
B

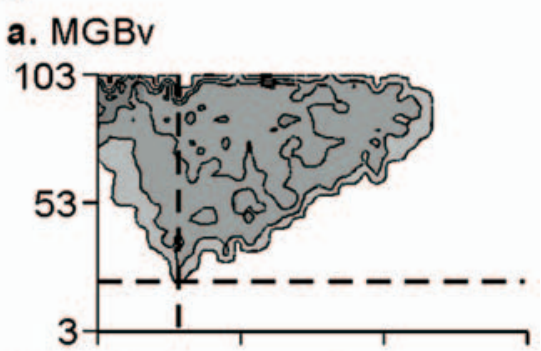

b. ICc before muscimol

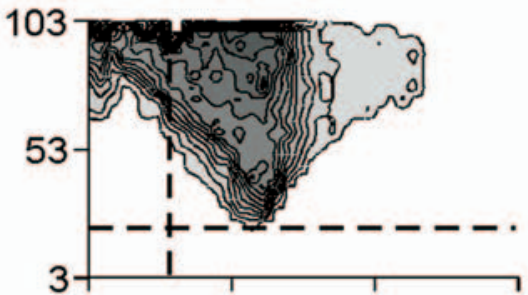

c. ICc after muscimol



d. ICC after ES

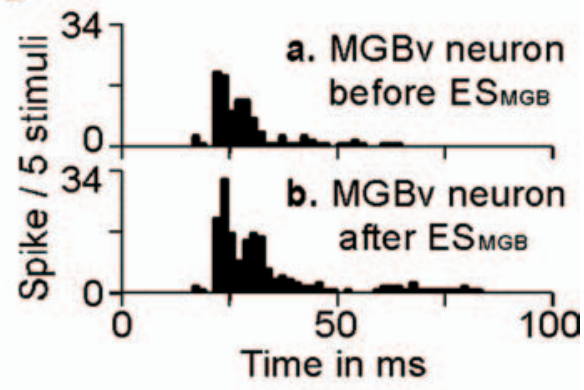

each frequency was defined as the boundary between two neighboring amplitudes in which one amplitude evoked more than one spike and the other evoked no response per 5 stimuli. If spontaneous activity interfered with spike counting, the threshold was determined by at least one spike difference between neighboring amplitudes, depending on the spontaneous rate. In this case, computer contour plotting was used. The starting point of the contour plotting was defined as being one spike above the level of spontaneous spikes. In addition, the thresholds were further adjusted and determined by tone-evoked responses shown in PST or cumulative PST histograms (Yan and Ehret, 2002; Yan and Zhang, 2005). The best frequency (BF), minimum threshold (MT), bandwidths at $10 \mathrm{~dB}$ (BW10) and $50 \mathrm{~dB}$ (BW50) above the MT, RF size, and average spike number (ASN) derived from the FA-scan data provided a comprehensive evaluation of RF plasticity. The BF was the single frequency at which a neuron showed the lowest response threshold (i.e., MT). The BW10 or BW50 was the frequency range at 10 or $50 \mathrm{~dB}$ above the $\mathrm{MT}$ at which a neuron showed excitatory responses to tone stimuli. The RF size was the number of frequency/amplitude sets within the $\mathrm{RF}<50 \mathrm{~dB}$ SPL where a neuron showed excitatory responses. The ASN was the total number of spikes in the RF divided by the number of frequency/amplitude sets within the RF. The spike number for each frequency/amplitude set was counted in a 60 -ms-wide window starting from the beginning of a PST histogram displaying responses to five identical stimuli. These ICc RF parameter values were compared before and after electrical stimulation of the MGBv.

Data was generally expressed by a mean \pm SD. A $t$ test was used to compare the differences between the two groups of data. A $p$ value of $<0.05$ was considered to be statistically significant for all analyses in this study.

\section{Results}

The effects of the $\mathrm{ES}_{\mathrm{MGB}}$ on ICc RFs were studied in 86 ICc neurons recorded from 22 mice in the saline group and in 32 ICc neurons recorded from eight mice in the muscimol group. Both $\mathrm{MGBv}$ and ICc neurons showed sharp frequency tunings and easily identifiable BFs and MTs. Their BFs ranged from 9 to $24 \mathrm{kHz}$ (14.76 \pm 3.58 $\mathrm{kHz}$ ) and their MTs ranged from 14 to 36 $\mathrm{dB}$ SPL $(23.4 \pm 7.3 \mathrm{~dB}$ SPL).

$\mathrm{ES}_{\mathrm{MGB}}$ with $700 \mathrm{nA}$ is efficient in inducing plastic changes in cortical RFs (Jafari et al., 2007). This current level is unlikely to damage the stimulated MGBv neurons, as shown in Figure $1 B$. This example illustrates that the stimulated MGBv neurons maintained a similar temporal response pattern but showed an increased response magnitude after $\mathrm{ES}_{\mathrm{MGB}}$. The increased response magnitude might result from the corticothalamic positive feedback (Yan and Suga, 1996; Yan and Ehret, 2002). ES- 
MGB consistently induced changes in the shape of ICc RFs regardless of whether saline solution was applied to the AI. The example in Figure $1 C$ represents a change in the ICc RF after $\mathrm{ES}_{\mathrm{MGB}}$. There were several notable differences between the stimulated MGBv $\mathrm{RF}$ and the recorded ICc RF; the MGBv neuron had a lower BF, lower MT, wider bandwidth, larger RF size, and lower ASN (Fig. $1 \mathrm{Ca}-\mathrm{Cc}$ ). Cortical application of saline itself did not alter the ICc RF (Fig. $1 C b, C c)$. $\mathrm{ES}_{\mathrm{MGB}}$ induced changes in ICc RF: a downward BF shift, MT decrease, bandwidth widening, RF expansion, and ASN decrease (Fig. $1 C c, C d$ ). These changes of ICc RFs were related to the differences in the parameter values between $\mathrm{MGBv}$ and ICc neurons and will be discussed below. The example in Figure $1 D$ shows that a cortical application of muscimol solution did not change the BF, but clearly elevated the MT of this ICc neuron (Fig. $1 D b, D c$ ) and blocked the development of the changes in the ICc RF (Fig. $1 D a, D c, D d$ ).

\section{Effects of $\mathrm{ES}_{\mathrm{MGB}}$ on collicular BFs with and without} cortical inactivation

Neurons at all levels of the central auditory pathway show tuning for sound frequency. Changes in the frequency tunings of auditory neurons have been the most common index for studying auditory plasticity. Therefore, we first focused on the analysis of the $\mathrm{ES}_{\mathrm{MGB}}$-evoked changes in the BFs of ICc neurons.

$\mathrm{ES}_{\mathrm{MGB}}$ caused robust $\mathrm{BF}$ shifts in a large population of the studied ICc neurons when saline was applied to the AI. The BF shifts could be either upward or downward. The average BF shift was insignificant $(15.14 \pm 3.54 \mathrm{kHz}$ before and $14.77 \pm 2.96 \mathrm{kHz}$ after $\left.\mathrm{ES}_{\mathrm{MGB}} ; n=86 ; p>0.05\right)$. Of 86 ICc neurons, the BFs of 34 $(39.5 \%)$ neurons shifted higher, those of 43 (50\%) neurons shifted lower and nine (10.5\%) neurons showed no BF shift. Because $90 \%$ of the ICc neurons showed BFs shift after $E_{M G B}$, we confirmed that the MGBv neurons impacted the processing of auditory information in the inferior colliculus. Although the data indicated that most neurons showed $\mathrm{BF}$ changes after $\mathrm{ES}_{\mathrm{MGB}}$, these percentages yielded little additional information.

We further observed that the direction of BF shifts was closely related to the difference in the BFs between the stimulated MGBv neurons and the recorded ICc neurons. The ICc neurons showed a downward BF shift when their BFs were higher than the BFs of the stimulated MGBv neurons. Conversely, the ICc neurons showed an upward BF shift when their BFs were lower than the BFs of the stimulated MGBv neurons. The BFs of ICc neurons changed little when the BF difference between MGBv and ICc neurons was within $\sim 2 \mathrm{kHz}$. Figure 2 shows the relationship of ICc BF shifts to the BF differences between the MGBv and ICc neurons. The larger the difference in BFs between the MGBv and ICc neurons, the larger the shift in BFs with a $-5 \mathrm{kHz}$ to $5 \mathrm{kHz}$ range of $\mathrm{BF}$ difference $(r=0.92)$. Beyond this range, the increase in BF shift decreased (Fig. 2, filled circles). The BF shifts of most ICc neurons almost entirely recovered $4 \mathrm{~h}$ after the $\mathrm{ES}_{\mathrm{MGB}}$. On average, the BF shifts recovered by $87.1 \pm 25.7 \%(n=77)$.

In $7(21.9 \%)$ of 32 ICc neurons in the muscimol group, $\mathrm{ES}_{\mathrm{MGB}}$ shifted the BFs either higher or lower by only $1 \mathrm{kHz}$. In addition, the BF changes in the muscimol group were apparently independent of the BF difference between the MGBv and ICc neurons (Fig. 2, open circles).

Effects of $\mathrm{ES}_{\mathrm{MGB}}$ on collicular MTs with and without cortical inactivation

When saline was applied to the $\mathrm{AI}$, the $\mathrm{ES}_{\mathrm{MGB}}$ did not significantly change the averaged ICc MTs $(23.67 \pm 6.38 \mathrm{~dB}$ SPL before and $24.12 \pm 5.95 \mathrm{~dB}$ SPL after $\left.\mathrm{ES}_{\mathrm{MGB}} ; n=86 ; p>0.05\right)$. However, the

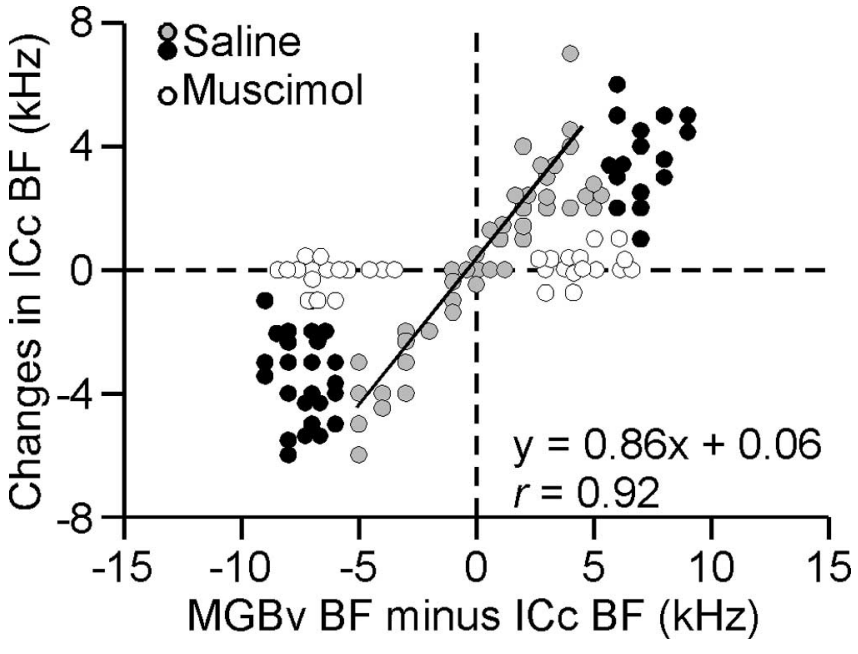

Figure 2. Effects of $E S_{M G B}$ on the BFs of ICC neurons. The ICC BF shift was systematically related to the difference in $B F$ between the stimulated $M G B v$ and recorded ICc neurons (gray circles). When the difference in BF was larger than $5 \mathrm{kHz}, \mathrm{ES}_{\mathrm{MGB}}$-evoked BF shift became smaller (black circles). A cortical application of muscimol abolished the $\mathrm{ES}_{\mathrm{MGB}}$-evoked ICC BF shift (white circles).

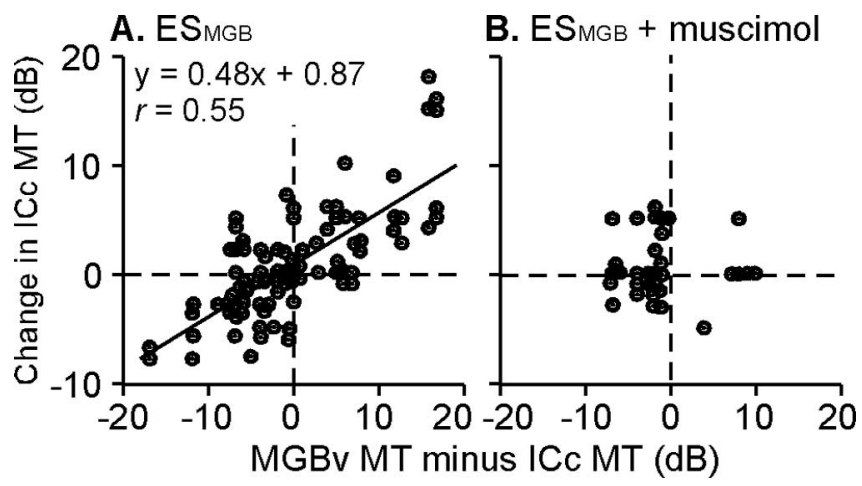

Figure 3. Effects of $E S_{M G B}$ on ICC MTs. $A$, Changes in ICC MTs were linearly related to the difference in MT between the stimulated MGBv and recorded ICC neurons. $\boldsymbol{B}$, This correlation disappeared when muscimol was applied to the AC.

MTs of individual neurons showed up to an $18 \mathrm{~dB}$ increase or decrease after $\mathrm{ES}_{\mathrm{MGB}}$. The ICc MTs systematically changed as a function of the MT difference between the MGBv and ICc neurons (Fig. 3A). The $\mathrm{ES}_{\mathrm{MGB}}$ increased ICc MTs when the MGBv MTs were higher than the ICc MTs whereas $\mathrm{ES}_{\mathrm{MGB}}$ decreased ICc MTs when the MGBv MTs were lower than the ICc MTs. The changes in ICc MTs displayed a linear correlation to the MT differences between the MGBv and ICc neurons $(n=86 ; r=$ $0.55)$. Four hours after the $\mathrm{ES}_{\mathrm{MGB}}$, the MT changes recovered by $69.6 \pm 27.2 \%(n=69)$.

When muscimol was applied to the auditory cortex, the MTs of $50 \%$ ( 16 of 32 ) of the ICc neurons increased by 5 or $10 \mathrm{~dB}$, the MTs of only $3.1 \%$ ( 1 of 32 ) of the neurons decreased by $5 \mathrm{~dB}$ and the MTs of $46.9 \%$ ( 15 of 32 ) of the neurons remained unchanged 5 min (the start time of the FA-scan) after the drug application. On average, a cortical application of muscimol significantly increased ICc MTs from $20.8 \pm 4.79 \mathrm{~dB}$ SPL to $23.6 \pm 3.56 \mathrm{~dB}$ SPL $(p<0.001)$. After muscimol inactivation of the $\mathrm{AI}$, the $\mathrm{ES}_{\mathrm{MGB}}$ changed the MTs of $13(40.6 \%)$ ICc neurons by $5 \mathrm{~dB}$; nine (28.1\%) neurons showed MT increases and four (12.5\%) neurons showed MT decreases. In contrast, the MTs of 19 (59.4\%) neurons were unchanged. Unlike those in the saline group, the 


\section{A. BW10}

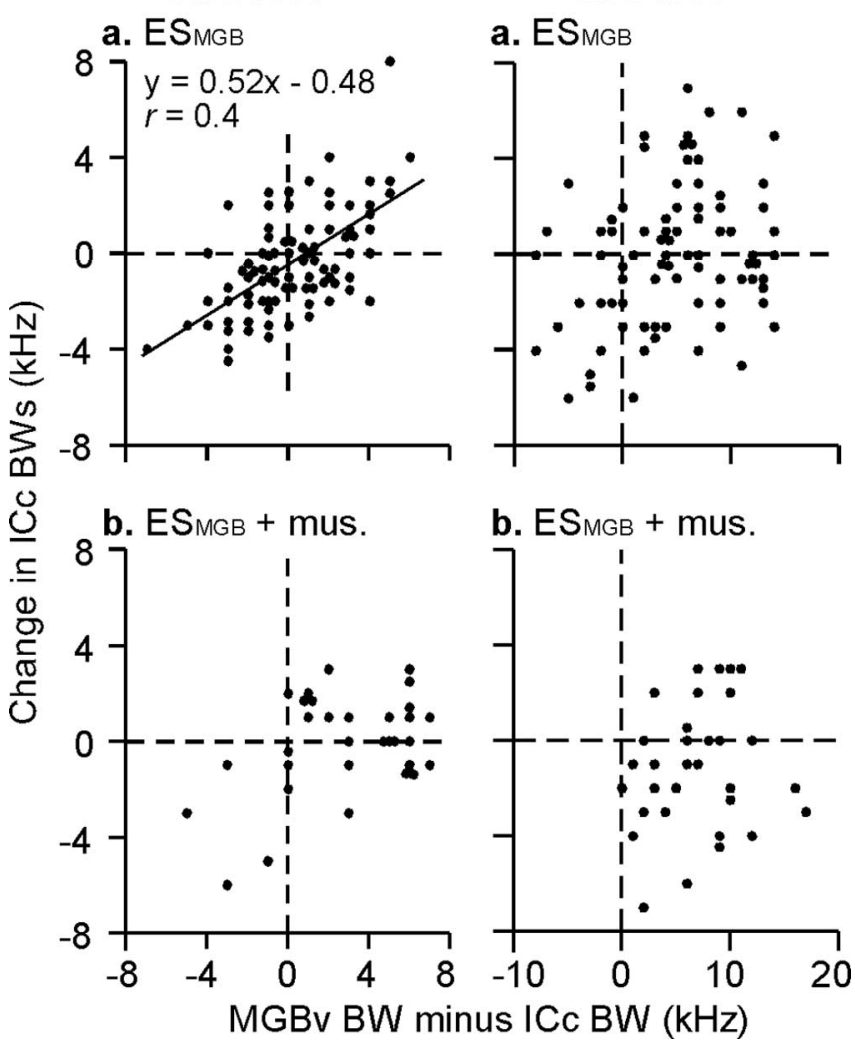

Figure 4. Effects of $E S_{M G B}$ on the bandwidths of ICC RFs. Aa, The changes in ICC BW10 were related to the difference in BW10 between the stimulated MGBv and recorded ICc neurons. $\boldsymbol{A} \boldsymbol{b}$, This correlation disappeared when muscimol solution was applied to the auditory cortex. $\mathbf{B a}$, $\boldsymbol{B} \boldsymbol{b}$, The changes in BW50 were poorly related to the difference in BW50 between the MGBv and ICc neurons. mus, Muscimol.

changes in the MTs of ICc neurons in the muscimol group were not correlated to the MT difference between MGBv and ICc neurons (Fig. 3B).

\section{Effects of $\mathrm{ES}_{\mathrm{MGB}}$ on collicular bandwidths with and without} cortical inactivation

The $\mathrm{ES}_{\mathrm{MGB}}$ either increased or decreased the bandwidths of the frequency-tuning curves of ICc neurons at $10 \mathrm{~dB}$ (BW10) and 50 $\mathrm{dB}$ (BW50) above the MTs when saline was applied to the AI. The average absolute change was $1.62 \pm 1.34 \mathrm{kHz}$ for BW10 and $2.16 \pm 1.87 \mathrm{kHz}$ for BW50 $(n=86)$. The change in BW50 was significantly larger than that in BW10 $(p<0.05)$. Although there were some variations, the $\mathrm{ES}_{\mathrm{MGB}}$ increased the BW10 s of ICc neurons when the BW10 s of stimulated MGBv neurons were wider than those of the recorded ICc neurons. However, $\mathrm{ES}_{\mathrm{MGB}}$ reduced the BW10 s of ICc neurons when the BW10 s of the stimulated MGBv neurons were narrower than those of the recorded ICc neurons. The changes in BW10 were correlated to the differences in BW10 between the MGBv and ICc neurons (Fig. $4 A a)(r=0.4)$. In contrast, the BW50 s appeared to be randomly altered by $\mathrm{ES}_{\mathrm{MGB}}$. The BW50 changes of ICc neurons were not related to the differences in BW50 between the MGBv and ICc neurons (Fig. $4 \mathrm{Ba})(r=0.07)$. Four hours after the $\mathrm{ES}_{\mathrm{MGB}}$, the changes recovered from by $61.8 \pm 32.8 \%(n=78)$ for BW10 and $59.6 \pm 27.2 \%(n=76)$ for BW50.

Cortical application of muscimol solution had little effect on ICc BW10, but had a significant impact on ICc BW50. Of 32 ICc neurons, BW10 was unchanged in 15 (46.9\%) neurons whereas

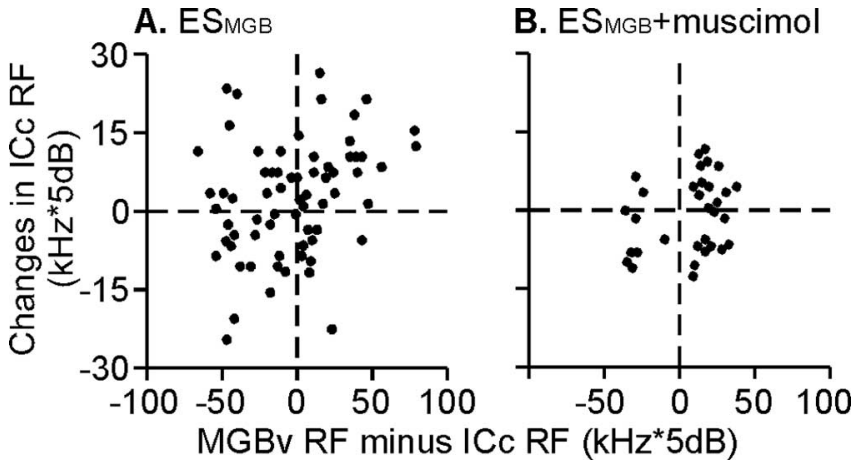

Figure 5. Effects of $E S_{M G B}$ on the RF size of ICc neurons. $A$, The changes in RF size evoked by $E_{M G B}$ were not related to the difference in RF size between the stimulated MGBv and recorded IC c neurons. $\boldsymbol{B}$, When muscimol was applied to the $A C$, these changes became smaller.

BW50 was unchanged in only $1(3.1 \%)$ neuron. On average, BW10 was $7.94 \pm 2.70 \mathrm{kHz}$ before and $8.00 \pm 2.71 \mathrm{kHz}$ after the cortical application of muscimol, which was of insignificant difference $(p>0.05)$. BW50 was $23.53 \pm 5.13 \mathrm{kHz}$ before and $21.25 \pm 5.81 \mathrm{kHz}$ after the cortical application of muscimol. Muscimol inactivation of the AI significantly reduced BW50 of ICc neurons $(p<0.01)$. After the cortical inactivation by muscimol, the $\mathrm{ES}_{\mathrm{MGB}}$ altered both BW10 and BW50. However, the changes in BW 10 or BW50 of ICc neurons were not related to the differences in BW10 or BW50 between the MGBv and ICc neurons (Figs. $4 A b, B b)$. BW10 increased in $14(43.8 \%)$ neurons and decreased in $13(40.6 \%)$ neurons whereas BW50 increased in 8 $(25.0 \%)$ neurons and decreased in $19(59.4 \%)$ neurons.

\section{Effects of $\mathrm{ES}_{\mathrm{MGB}}$ on collicular RF sizes with and without} cortical inactivation

Similar to the changes in BW50, the $\mathrm{ES}_{\mathrm{MGB}}$ either increased or decreased the RF size of ICc neurons when saline was applied to the AI. However, the average RF size was unchanged (i.e., $59.30 \pm$ $15.81 \mathrm{kHz} \times 5 \mathrm{~dB}$ before $\mathrm{ES}_{\mathrm{MGB}}$ vs $56.94 \pm 17.07 \mathrm{kHz} \times 5 \mathrm{~dB}$ after $\left.\mathrm{ES}_{\mathrm{MGB}} ; n=86 ; p>0.05\right)$. The $\mathrm{ES}_{\mathrm{MGB}^{-}}$-evoked changes in $\mathrm{RF}$ size were not related to the differences in RF size between the MGBv and ICc neurons ( $r=0.06$ ) (Fig. 5A). Four hours after the $\mathrm{ES}_{\mathrm{MGB}}$, the changes in RF size recovered from their peak values by $57.5 \pm 31.5 \%(n=81)$. In the muscimol group, a cortical application of muscimol significantly reduced the RF size of ICc neurons from $61.69 \pm 17.64 \mathrm{kHz} \times 5 \mathrm{~dB}$ to $54.16 \pm 17.67 \mathrm{kHz} \times 5 \mathrm{~dB}$ $(n=32 ; p<0.01)$. Based on counts of single neurons, $71.9 \%$ (23 of 32) of the neurons showed a decrease, $25.0 \%$ ( 8 of 32) showed an increase, and only $3.1 \%$ ( 1 of 32 ) showed no change in their RF size. Furthermore, the $\mathrm{ES}_{\mathrm{MGB}}$ did not significantly change the RF size of ICc neurons (i.e., $57.88 \pm 17.67 \mathrm{kHz} \times 5 \mathrm{~dB}$ vs $54.16 \pm$ $13.78 \mathrm{kHz} \times 5 \mathrm{~dB} ; p>0.05)$. The changes in the RF size of individual neurons were not correlated to the differences in RF size between the MGBv and ICc neurons (Fig. 5B).

Effects of $\mathrm{ES}_{\mathrm{MGB}}$ on collicular ASN with and without cortical inactivation

We used the ASN to evaluate the overall magnitude of excitatory responses of auditory neurons to various tone frequencies and amplitudes $<50 \mathrm{~dB}$ SPL. The average MGBv ASN was $25.76 \pm$ $11.77(n=22)$ and the average ICc ASN was $28.93 \pm 15.81(n=$ $86)$. There was no statistical difference between them $(p>0.05)$. When saline was applied to the $\mathrm{AI}$, the $\mathrm{ES}_{\mathrm{MGB}}$ significantly reduced the ICc ASN from $28.93 \pm 15.81-22.71 \pm 12.27(n=86$, $p<0.01)$. However, the ASNs of individual neurons could be 


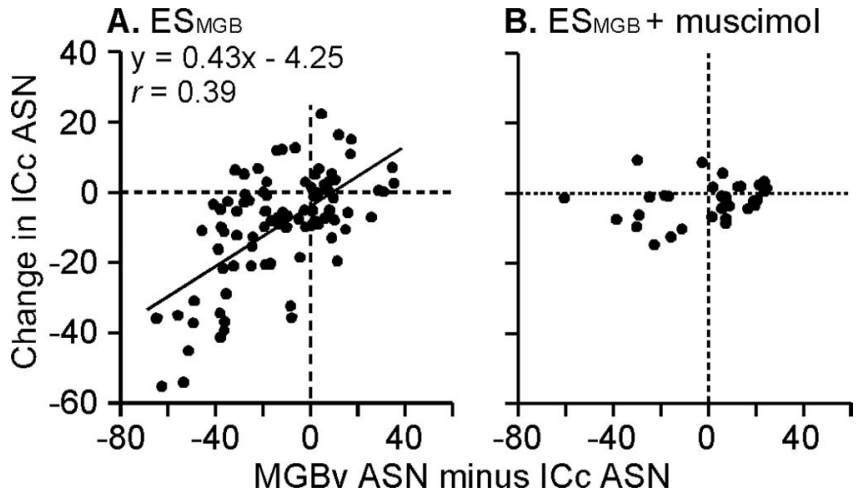

Figure 6. Effects of $E S_{M G B}$ on ICC ASNs. $A$, The changes in ASNs evoked by $E S_{M G B}$ were related to the difference in ASN between the stimulated MGBv and recorded ICc neurons. $\boldsymbol{B}$, This correlation disappeared when muscimol was applied to the AC.

either increased or decreased by $\mathrm{ES}_{\mathrm{MGB}}$; the changes were not random, but correlated to the differences in ASNs between MGBv and ICc neurons $(r=0.39)$. The ICc ASN showed a tendency to increase when MGBv ASN was higher than ICc ASN whereas it showed a tendency to decrease when the MGBv ASN was lower than the ICc ASN. The function of the ICc ASN change against the ASN difference shifted downward by 4.25 (Fig. 6A). Four hours after the $\mathrm{ES}_{\mathrm{MGB}}$, the ASN changes recovered by $48.4 \pm$ $29.1 \%(n=77)$.

In the muscimol group, the control ASN of ICc neurons was $27.46 \pm 19.75(n=32)$. Cortical application of muscimol solution significantly reduced it to $21.03 \pm 12.50(p<0.05)$. $\mathrm{ES}_{\mathrm{MGB}}$ had no additional impact on ICc ASN $(21.85 \pm 15.98 ; p>0.05)$, although the ASNs of some ICc neurons could increase or decrease. Additionally, the changes in the ASNs of individual ICc neurons were not related to the ASN differences between the MGBv and ICc neurons (Fig. 6B).

\section{Changes in measured parameters without cortical inactivation and $\mathrm{ES}_{\mathrm{MGB}}$}

The stability of our recordings was examined in the RFs of 12 ICc neurons of three mice over a $5 \mathrm{~h}$ recording period. In each neurons, the RF recorded first was used as the control. Then, the neuronal RF was recorded at every $30 \mathrm{~min}$. The largest changes in BF, MT, BW10, BW50, RF size, and ASN over the 5 h observation period were selected to evaluate RF fluctuations. Their designations were different from those of the control RF.

The BFs of the ICc neurons were very stable. None of the 12 neurons showed changes in BF over the $5 \mathrm{~h}$ observation period. The MT, bandwidth, RF size, and ASN of some neurons, however, showed small fluctuations. On average, the changes were $2.92 \pm 3.97 \mathrm{~dB}$ for MT, $1.25 \pm 1.87 \mathrm{kHz}$ for BW10, $0.92 \pm 2.84$ $\mathrm{kHz}$ for BW50, $-3.58 \pm 4.25 \mathrm{kHz} \times 5 \mathrm{~dB}$ for $\mathrm{RF}$ size, and $-0.77 \pm 10.71$ for ASN. These data confirm the stability of our recordings.

\section{Discussion}

Potential pathways involved in the collicular plasticity evoked by thalamic stimulation

In the lemniscal ascending pathway, neural inputs to the inferior colliculus are the most complex. The ascending inputs consist of bilateral projections from all subcollicular nuclei whereas the ascending outputs of the inferior colliculus are quite simple (i.e., ipsilateral projections to the medial geniculate body of the thalamus) (Oliver and Huerta, 1992; Pollak et al., 2003). This unique anatomical feature allows the inferior colliculus to function both as a convergence/integration center and as a preprocessor for advanced computation in the auditory thalamus and cortex.

The ICc receives descending inputs from the auditory thalamus and cortex (i.e., corticocollicular and thalamocollicular inputs) (Huffman and Henson, 1990; Winer et al., 1998). The point-to-point tonotopy is a predominant feature of the colliculothalamic and thalamocortical projections as well as of the corticothalamic and cortiocollicular projections (Redies et al., 1989; Saldana et al., 1996; Winer et al., 1999, 2001; Bajo and Moore, 2005; Takayanagi and Ojima, 2006; Bajo et al., 2007; Lim and Anderson, 2007). The colliculothalamic, thalamocortical, and corticofugal projections constitute reciprocal connections that link the inferior colliculus, the medial geniculate body, and the auditory cortex in a tonotopic loop [colliculo-thalamo-corticocollicular loop (CTCC loop)]. This tonotopic feature suggests that the CTCC loop is a strong candidate for mediating the highly specific changes in ICc RFs evoked by the $\mathrm{ES}_{\mathrm{MGB}}$ as observed in current study. In addition, the existence of direct thalamocollicular projections presents another possibility (Adams, 1980; Frisina et al., 1997; Kuwabara and Zook, 2000; Winer et al., 2002). The potential role of the CTCC loop and thalamocollicular projections in thalamocollicular modulation is discussed below.

\section{CTCC loop for sound-specificity of learning-induced and experience-dependent auditory plasticity}

The thalamocortical projections, the final pathway to the auditory cortex, appear to be particularly important in the CTCC loop. The construction of cortical receptive fields, however, is determined by thalamocortical projections (Miller et al., 2001; Suder et al., 2002; Metherate et al., 2005). Our previous study has shown that focal thalamic stimulation systematically changes the BF, MT, bandwidth, RF size, and ASN of cortical neurons toward those of the stimulated thalamic neurons (Jafari et al., 2007). This suggests that the thalamocortical system harbors an intrinsic mechanism for sound-specific auditory plasticity.

Corticofugal modulation has been extensively explored for the past decade. Focal cortical stimulation results in featurespecific changes in subcortical RFs (J. Yan and N. Suga, 1996; W. Yan and N. Suga, 1998; Zhou and Jen, 2000, 2005; Ma and Suga, 2001a,b, 2007; Yan and Ehret, 2002; Jen and Zhou, 2003; Yan et al., 2005;). Apparently, corticofugal projections are an important neural substrate for neural plasticity in the subcortical auditory nuclei. It has been documented that auditory learning induces the shifts of cortical and subcortical RFs toward the frequency of the learned tone (Diamond and Weinberger, 1986; Gonzalez-Lima and Agudo, 1990; Lennartz and Weinberger, 1992; Gao and Suga, 1998, 2000; Ji and Suga, 2003). A previous study of the inferior colliculus of bats validates that learning-induced RF plasticity is largely reduced when the auditory cortex is treated with atropine, a muscarinic acetylcholine receptor antagonist (Ji et al., 2001).

In mice, electrical stimulation of the cholinergic basal forebrain paired with a tone shifts the BFs of cortical and collicular neurons toward the frequency of the paired tone. Cortical application of atropine or muscimol abolishes the plastic changes in both the auditory cortex and the inferior colliculus (Yan and Zhang, 2005; Zhang et al., 2005). We also found that basal forebrain stimulation paired with a tone induces frequency-specific plastic changes in the medial geniculate body; the development of the thalamic plastic can also be abolished by cortical inactivation (unpublished data). These findings establish that the learninginduced or experience-dependent plasticity of collicular receptive fields is widely dependent on the corticofugal system. 
If the CTCC loop does function in an integrated manner, the plasticity of cortical and collicular RFs should be similar to each other after focal thalamic stimulation. As expected, the RF plasticity of collicular neurons was very similar to that of cortical neurons. Namely, the BF (Fig. 2A), MT (Fig. 3A), BW10 (Fig. $4 A$ ), and ASN (Fig. $6 A$ ) of the collicular receptive field showed systematic changes toward those of the receptive fields of the stimulated neuron. These plastic changes were eliminated when the auditory cortex was inactivated by muscimol. Apparently, the CTCC loop conveys the thalamic information to the inferior colliculus. Our findings support previous findings in bats where focal stimulation of collicular neurons evoked shift of the frequency tuning curves of neighboring collicular neurons toward those of the stimulated neurons through the corticocollicular feedback (Zhang and Suga, 2005). Therefore, our data suggest that the tonotopic CTCC loop coordinates the sound-specific plasticity in the inferior colliculus, the medial geniculate body, and the auditory cortex.

\section{Possible role of thalamocollicular projection}

Anatomic studies also demonstrate that the inferior colliculus receives ipsilateral descending inputs from the medial geniculate body (Adams, 1980; Frisina et al., 1997; Kuwabara and Zook, 2000; Winer et al., 2002). A question is to what extent the thalamocollicular projection is involved in the collicular RF plasticity evoked by $\mathrm{ES}_{\mathrm{MGB}}$ although the number of thalamocollicular fibers is much less than that of corticocollicular fibers. Compared with the $\mathrm{ES}_{\mathrm{MGB}}$-evoked cortical RF plasticity (Jafari et al., 2007),

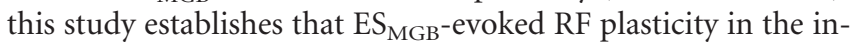
ferior colliculus predominately inherits the plasticity of the auditory cortex through the corticocollicular projections. $\mathrm{ES}_{\mathrm{MGB}}$ did not induce any significant changes in the RFs of collicular neurons when the auditory cortex was inactivated by muscimol. However, certain parameter values of the receptive fields of individual collicular neurons showed random deviation from their original values. Compared with the normal fluctuations of collicular RFs, the deviations found in BW10, BW50, and RF size were considerably larger (Figs. $4 A b, B b, 5 B$ ). The changes in bandwidth and RF size induced by $\mathrm{ES}_{\mathrm{MGB}}$ under cortical inactivation could be attributable to thalamocollicular modulation. It should be noted that these changes may also reflect the decreased thalamic activity that occurs through corticothalamic feedback when the auditory cortex is inactivated by muscimol (Zhang and Suga, 1997). The function of thalamocollicular projections remains to be studied.

\section{Tonic influence of the auditory cortex on the inferior colliculus}

Muscimol causes complete and reversible inhibition of the activities of cortical neurons when applied to the cortex (Reiter and Stryker, 1988; Kasamatsu et al., 2005). It is also known that cortical application of muscimol significantly reduces the responses of subcortical neurons to acoustic stimuli (Zhang and Suga, 1997; Yan and Suga, 1999; Zhang et al., 2005). Consistent with previous reports, we showed in this study that the auditory responses and receptive fields of collicular neurons are significantly reduced after cortical application of muscimol solution; it suggests an effective inactivation of the auditory cortex.

The general effect of the corticofugal projections (i.e., whether they are inhibitory, excitatory or both) is controversial (Watanabe et al., 1966; Amato et al., 1969; Syka and Popelar, 1984; Sun et al., 1989). Some studies document that the corticofugal effects are generally excitatory (Villa et al., 1991; Zhang and Suga,
1997; Yan and Suga, 1999) whereas others indicate that the corticofugal effects are both inhibitory and excitatory (Ryugo and Weinberger, 1976; Syka and Popelar, 1984; Popelar et al., 2003). Our current study analyzed the effects of cortical inactivation on a number of parameters including the BF, MT, bandwidth, RF size, and ASN of collicular neurons. We showed that the inactivation of the auditory cortex did not alter collicular BFs, but that it significantly altered the other four parameters. Of 32 collicular neurons studied, the MTs of $50.0 \%$ of the neurons increased and the RF size of $71.9 \%$ of the neurons decreased after cortical inactivation. On average, cortical inactivation also significantly reduced BW50 and ASN. Therefore, our data generally supports the conclusion that corticofugal influence is excitatory despite the observation that a few collicular neurons changed in an opposite manner after cortical inactivation with muscimol.

\section{References}

Adams JC (1980) Crossed and descending projections to the inferior colliculus. Neurosci Lett 19:1-5.

Amato G, La Grutta V, Enia F (1969) The control exerted by the auditory cortex on the activity of the medial geniculate body and inferior colliculus. Arch Sci Biol (Bologna) 4:291-313.

Bajo VM, Moore DR (2005) Descending projections from the auditory cortex to the inferior colliculus in the gerbil, Meriones unguiculatus. J Comp Neurol 486:101-116.

Bajo VM, Nodal FR, Bizley JK, Moore DR, King AJ (2007) The ferret auditory cortex: descending projections to the inferior colliculus. Cereb Cortex 17:475-491.

Diamond DM, Weinberger NM (1986) Classical conditioning rapidly induces specific changes in frequency receptive fields of single neurons in secondary and ventral ectosylvian auditory cortical fields. Brain Res 372:357-360.

Franklin, KBJ, Paxinos, G (1996) The mouse brain in stereotaxic coordinates. San Diego: Academic.

Frisina RD, Walton JP, Lynch-Armour MA, Klotz D (1997) Efferent projections of a physiologically characterized region of the inferior colliculus of the young adult CBA mouse. J Acoust Soc Am 101:2741-2753.

Gao E, Suga N (1998) Experience-dependent corticofugal adjustment of midbrain frequency map in bat auditory system. Proc Natl Acad Sci USA 95:12663-12670.

Gao E, Suga N (2000) Experience-dependent plasticity in the auditory cortex and the inferior colliculus of bats: role of the corticofugal system. Proc Natl Acad Sci USA 97:8081-8086.

Gonzalez-Lima F, Agudo J (1990) Functional reorganization of neural auditory maps by differential learning. NeuroReport 1:161-164.

Huffman RF, Henson Jr OW (1990) The descending auditory pathway and acousticomotor system: connections with the inferior colliculus. Brain Res Rev 15:295-323.

Jafari MR, Zhang Y, Yan J (2007) Multiparametric changes in the receptive field of cortical auditory neurons induced by thalamic activation in the mouse. Cereb Cortex 17:71-80.

Jen PH, Zhou X (2003) Corticofugal modulation of amplitude domain processing in the midbrain of the big brown bat, Eptesicus fuscus. Hear Res 184:91-106.

Ji W, Suga N (2003) Development of reorganization of the auditory cortex caused by fear conditioning: effect of atropine. J Neurophysiol 90:1904-1909.

Ji W, Gao E, Suga N (2001) Effect of acetylcholine and atropine on plasticity of central auditory neurons caused by conditioning in bats. J Neurophysiol 86:211-225.

Kasamatsu T, Mizobe K, Sutter EE (2005) Muscimol and baclofen differentially suppress retinotopic and nonretinotopic responses in visual cortex. Vis Neurosci 22:839-858.

Kuwabara N, Zook JM (2000) Geniculo-collicular descending projections in the gerbil. Brain Res 878:79-87.

Lennartz RC, Weinberger NM (1992) Frequency-specific receptive field plasticity in the medial geniculate body induced by pavlovian fear conditioning is expressed in the anesthetized brain. Behav Neurosci 106:484-497.

Lim HH, Anderson DJ (2007) Antidromic activation reveals tonotopically 
organized projections from primary auditory cortex to the central nucleus of the inferior colliculus in guinea pig. J Neurophysiol 97:1413-1427.

Ma X, Suga N (2001a) Plasticity of bat's central auditory system evoked by focal electric stimulation of auditory and/or somatosensory cortices. J Neurophysiol 85:1078-1087.

Ma X, Suga N (2001b) Corticofugal modulation of duration-tuned neurons in the midbrain auditory nucleus in bats. Proc Natl Acad Sci USA 98:14060-14065.

Ma X, Suga N (2003) Augmentation of plasticity of the central auditory system by the basal forebrain and/or somatosensory cortex. J Neurophysiol 89:90-103.

MaX, Suga N (2007) Multiparametric corticofugal modulation of collicular duration-tuned neurons: modulation in the amplitude domain. J Neurophysiol 97:3722-3730.

Metherate R, Kaur S, Kawai H, Lazar R, Liang K, Rose HJ (2005) Spectral integration in auditory cortex: mechanisms and modulation. Hear Res 206:146-158

Miller LM, Escabi MA, Read HL, Schreiner CE (2001) Functional convergence of response properties in the auditory thalamocortical system. Neuron 32:151-160.

Oliver DL, Huerta MF (1992) Inferior and superior colliculus. In: The mammalian auditory pathways: neuroanatomy (Webster DB, Popper AN, Fay RR, eds), pp 168-221. Springer, New York.

Pollak GD, Burger RM, Klug A (2003) Dissecting the circuitry of the auditory system. Trends Neurosci 26:33-39.

Popelar J, Nwabueze-Ogbo FC, Syka J (2003) Changes in neuronal activity of the inferior colliculus in rat after temporal inactivation of the auditory cortex. Physiol Res 5:615-628.

Redies H, Brandner S, Creutzfeldt OD (1989) Anatomy of the auditory thalamocortical system of the guinea pig. J Comp Neurol 282:489-511.

Reiter HO, Stryker MP (1988) Neural plasticity without postsynaptic action potentials: Less-active inputs become dominant when kitten visual cortical cells are pharmacologically inhibited. Proc Natl Acad Sci USA 85:2623-2627.

Ryugo DK, Weinberger NM (1976) Corticofugal modulation of the medial geniculate body. Exp Neurol 2:77-391.

Saldana E, Feliciano M, Mugnaini E (1996) Distribution of descending projections from primary auditory neocortex to inferior colliculus mimics the topography of intracollicular projections. J Comp Neurol 371(1):15-40.

Suder K, Funke K, Zhao Y, Kerscher N, Wennekers T, Worgotter F (2002) Spatial dynamics of receptive fields in cat primary visual cortex related to the temporal structure of thalamocortical feedforward activity. Experiments and models. Exp Brain Res 144:430-444.

Suga N, MaX (2003) Multiparametric corticofugal modulation and plasticity in the auditory system. Nat Rev Neurosci 4:783-794.

Suga N, Xiao Z, Ma X, Ji W (2002) Plasticity and corticofugal modulation for hearing in adult animals. Neuron 36:9-18.

Sun XD, Jen PH, Sun DX, Zhang SF (1989) Corticofugal influences on the responses of bat inferior collicular neurons to sound stimulation. Brain Res 1:1-8.

Syka J, Popelar J (1984) Inferior colliculus in the rat: neuronal responses to stimulation of the auditory cortex. Neurosci Lett 2:235-240.
Takayanagi M, Ojima H (2006) Microtopography of the dual corticothalamic projections originating from domains along the frequency axis of the cat primary auditory cortex. Neuroscience 142:769-780.

Villa AE, Rouiller EM, Simm GM, Zurita P, de Ribaupierre Y, de Ribaupierre F (1991) Corticofugal modulation of the information processing in the auditory thalamus of the cat. Exp Brain Res 3:506-517.

Watanabe T, Yanagisawa K, Kanzaki J, Katsuki Y (1966) Cortical efferent flow influencing unit responses of medial geniculate body to sound stimulation. Exp Brain Res 4:302-317.

Weinberger NM (1998) Physiological memory in primary auditory cortex: characteristics and mechanisms. Neurobiol Learn Mem 70:226-251.

Winer JA, Larue DT, Diehl JJ, Hefti BJ (1998) Auditory cortical projections to the cat inferior colliculus. J Comp Neurol 400:147-174.

Winer JA, Sally SL, Larue DT, Kelly JB (1999) Origins of medial geniculate body projections to physiologically defined zones of rat primary auditory cortex. Hear Res 130:42-61.

Winer JA, Diehl JJ, Larue DT (2001) Projections of auditory cortex to the medial geniculate body of the cat. J Comp Neurol 430:27-55.

Winer JA, Chernock ML, Larue DT, Cheung SW (2002) Descending projections to the inferior colliculus from the posterior thalamus and the auditory cortex in rat, cat, and monkey. Hear Res 168:181-195.

Yan J (2003) Canadian association of neuroscience review: development and plasticity of the auditory cortex. Can J Neurol Sci 30:189-200.

Yan J, Ehret G (2002) Corticofugal modulation of midbrain sound processing in the house mouse. Eur J Neurosci 16:119-128.

Yan J, Suga N (1996) Corticofugal modulation of time-domain processing of biosonar information in bats. Science 273:1100-1103.

Yan J, Suga N (1999) Corticofugal amplification of facilitative auditory responses of subcortical combination-sensitive neurons in the mustached bat. J Neurophysiol 2:817-824.

Yan J, Zhang Y (2005) Sound-guided shaping of the receptive field in the mouse auditory cortex by basal forebrain activation. Eur J Neurosci 21:563-576.

Yan J, Zhang Y, Ehret G (2005) Corticofugal shaping of frequency tuning curves in the central nucleus of the inferior colliculus of mice. J Neurophysiol 93:71-83.

Yan W, Suga N (1998) Corticofugal modulation of the midbrain frequency map in the bat auditory system. Nat Neurosci 1:54-58.

Zhang Y, Suga N (1997) Corticofugal amplification of subcortical responses to single tone stimuli in the mustached bat. J Neurophysiol 6:3489-3492.

Zhang Y, Suga N (2005) Corticofugal feedback for collicular plasticity evoked by electric stimulation of the inferior colliculus. J Neurophysiol 94:2676-2682.

Zhang Y, Hakes JJ, Bonfield SP, Yan J (2005) Corticofugal feedback for auditory midbrain plasticity elicited by tones and electrical stimulation of basal forebrain in mice. Eur J Neurosci 22:871-879.

Zhou X, Jen PH (2000) Brief and short-term corticofugal modulation of subcortical auditory responses in the big brown bat, Eptesicus fuscus. J Neurophysiol 84:3083-3087.

Zhou X, Jen PH (2005) Corticofugal modulation of directional sensitivity in the midbrain of the big brown bat, Eptesicus fuscus. Hear Res 203:201-215. 